\title{
Manejo nutricional e farmacológico da obesidade pediátrica: um tratamento multiprofissional
}

\author{
Nutritional and pharmacological management of pediatric obesity: a multiprofessional treatment \\ Tratamiento nutricional y farmacológico de la obesidad pediátrica: un tratamiento multiprofisional
}

Recebido: 08/01/2021 | Revisado: 18/01/2021 | Aceito: 25/01/2021 | Publicado: 27/01/2021

\author{
Anaís Bezerra de Gusmão \\ ORCID: https://orcid.org/0000-0001-7170-9410 \\ Residência Multiprofissional em Saúde da Criança, Brasil \\ E-mail: anaisgusmao@gmail.com \\ Sara de Sousa Rocha \\ ORCID: https://orcid.org/0000-0002-7687-3575 \\ Residência Multiprofissional em Saúde da Criança, Brasil \\ E-mail: nutricionistasararocha@gmail.com \\ Bruno Wesley Ramalho Cirilo Ferreira \\ ORCID: https://orcid.org/0000-0003-3448-0957 \\ Residência Multiprofissional em Saúde da Criança, Brasil \\ E-mail:brunnoramallho@hotmail.com \\ Aline Kely Felício de Sousa Santos \\ ORCID: https://orcid.org/0000-0002-2720-3129 \\ Universidade Federal da Paraíba, Brasil \\ E-mail: alinekely.f@gmail.com \\ Cibério Landim Macedo \\ ORCID: https://orcid.org/0000-0002-0824-4056 \\ Residência Multiprofissional em Saúde da Criança, Brasil \\ E-mail:ciberiolandim@hotmail.com
}

\begin{abstract}
Resumo
A obesidade pediátrica, quando não tratada, tende a gerar diversas complicações que podem se estender até a vida adulta. Seu manejo adequado possibilita bons resultados, proporcionando melhor qualidade de vida aos pacientes. Neste contexto, o objetivo deste estudo foi investigar as principais terapias nutricionais e farmacológicas utilizadas nessa condição. Foi realizada uma revisão sistemática da literatura, baseando-se na busca de artigos científicos indexados no PubMed, ScienceDirect e Scielo, utilizando como descritores: obesidade pediátrica, tratamento, sibutramina, orlistate, liraglutida e aleitamento materno, bem como suas traduções para o inglês. Hábitos alimentares saudáveis, associados à prática de exercícios físicos, são consideradas estratégias eficazes de tratamento, até mesmo para casos de obesidade grave, sendo o acompanhamento nutricional bem estruturado indispensável para o sucesso terapêutico. Já os medicamentos antiobesidade são recursos utilizados quando apenas as mudanças no estilo de vida não são suficientes para atingir resultados satisfatórios. De acordo com a Sociedade Brasileira de Pediatria, as possibilidades farmacoterapêuticas mais recomendadas são sibutramina, orlistate e liraglutida. Todavia, esses agentes, apesar de desencadearem efeitos benéficos, podem gerar eventos adversos, sendo necessário realizar o acompanhamento constante dos pacientes. Assim, é de extrema importância que o prescritor faça uma avaliação rigorosa dos riscos e benefícios proporcionados pela farmacoterapia antiobesidade disponível, além de atividades benéficas desenvolvidas por parte da equipe multidisciplinar de saúde pelas melhorias nos hábitos da criança, visando a manutenção dos resultados a longo prazo.
\end{abstract}

Palavras-chave: Obesidade pediátrica; Dietoterapia; Tratamento farmacológico.

\begin{abstract}
Pediatric obesity, when left untreated, tends to generate several complications that can extend into adulthood. Proper management allows good results, providing better quality of life to patients. In this context, the objective of this study was to investigate the main nutritional and pharmacological therapies used in this condition. A systematic review of the literature was carried out, based on the search for scientific articles indexed in PubMed, ScienceDirect and Scielo, using the following descriptors: pediatric obesity, treatment, sibutramine, orlistate, liraglutide and breastfeeding, as well as their translations into English. Healthy eating habits, associated with the practice of physical exercises, are considered effective treatment strategies, even for cases of severe obesity, and well-structured nutritional monitoring is indispensable for therapeutic success. Anti-obesity drugs, on the other hand, are resources used when changes in lifestyle alone are not sufficient to achieve satisfactory results. According to the Brazilian Society of Pediatrics, the most recommended pharmacotherapeutic possibilities are sibutramine, orlistat and liraglutide. However, these agents,
\end{abstract}


despite triggering beneficial effects, can generate adverse events, requiring the constant monitoring of patients. Thus, it is extremely important that the prescriber makes a rigorous assessment of the risks and benefits provided by the available anti-obesity pharmacotherapy, in addition to beneficial activities developed by the multidisciplinary health team for the improvement of the child's habits, aiming at maintaining long-term results.

Keywords: Pediatric obesity; Diet Therapy; Pharmacological treatment.

\section{Resumen}

La obesidad pediátrica, cuando no se trata, tiende a generar varias complicaciones que pueden extenderse hasta la edad adulta. Un manejo adecuado permite buenos resultados, brindando una mejor calidad de vida a los pacientes. En este contexto, el objetivo de este estudio fue investigar las principales terapias nutricionales y farmacológicas utilizadas en esta patología. Se realizó una revisión sistemática de la literatura a partir de la búsqueda de artículos científicos indexados en PubMed, ScienceDirect y Scielo, utilizando los siguientes descriptores: obesidad pediátrica, tratamiento, sibutramina, orlistato, liraglutida y lactancia materna, así como sus traducciones al inglés. Los hábitos alimentarios saludables, asociados a la práctica de ejercicios físicos, se consideran estrategias de tratamiento eficaces, incluso en los casos de obesidad severa, y un seguimiento nutricional bien estructurado es indispensable para el éxito terapéutico. Los medicamentos contra la obesidad, por otro lado, son recursos que se utilizan cuando los cambios en el estilo de vida por sí solos no son suficientes para lograr resultados satisfactorios. Según la Sociedad Brasileña de Pediatría, las posibilidades farmacoterapéuticas más recomendadas son sibutramina, orlistat y liraglutida. Sin embargo, estos agentes, a pesar de desencadenar efectos beneficiosos, pueden generar eventos adversos, requiriendo el seguimiento constante de los pacientes. Por ello, es de suma importancia que el prescriptor realice una evaluación rigurosa de los riesgos y beneficios que aporta la farmacoterapia antiobesidad disponible, además de las actividades beneficiosas desarrolladas por el equipo multidisciplinar de salud para la mejora de los hábitos del niño, con el objetivo de mantener resultados a largo plazo.

Palabras clave: Obesidad pediátrica; Terapia dietética; Tratamiento farmacológico.

\section{Introdução}

A obesidade pediátrica é uma doença multissistêmica que pode ocasionar complicações a curto, médio e longo prazo, como diabetes mellitus tipo 2, hipertensão arterial sistêmica, puberdade precoce, alterações menstruais, esteato-hepatite, distúrbios psicológicos, dentre outros (Rajjo et al., 2017). Além disso, estudos apontam que quatro em cada cinco adolescentes com obesidade continuam a ter problemas de peso quando adultos, aumentando a gravidade dos riscos futuros à saúde (Chao, Wadden, \& Berkowitz, 2018).

O sobrepeso e a obesidade, que antes eram desordens mais presentes em países de alta renda, estão aumentando em outros cenários, principalmente em áreas urbanas de países com baixa renda. Dados de 2019 revelam que cerca de 38 milhões de crianças com menos de 5 anos apresentavam sobrepeso ou obesidade. Adicionalmente, mais de 340 milhões de crianças e adolescentes de 5 a 19 anos estavam acima do peso ou obesas em 2016. Esse número corresponde a um aumento considerável, indicando que a prevalência de sobrepeso e obesidade entre crianças e adolescentes nessa faixa etária passou de 4\%, em 1975, para 18\%, em 2016 (World Health Organization, 2020).

No Brasil, os dados mostram que entre as crianças menores de 5 anos, 15,9\% têm excesso de peso. Além disso, três em cada dez crianças entre 5 e 9 anos estão acima do peso e, na adolescência, as taxas equivalem a 20,5\% (Brasil, 2020a; Brasil, 2018). Portanto, é necessário encontrar tratamentos que sejam seguros e eficazes na obesidade pediátrica, visando benefícios para a saúde e o bem-estar desses pacientes (Chao et al., 2018).

O manejo da obesidade é composto por diferentes medidas multidisciplinares, podendo incluir intervenções comportamentais, nutricionais, farmacológicas e cirúrgicas (Rajjo et al., 2017). As mudanças na dieta e a prática de exercícios físicos, além de alterações comportamentais, são consideradas como os principais tratamentos, até mesmo para jovens com obesidade grave. Entretanto, a perda de peso clinicamente considerável, bem como a sua manutenção, por meio de programas de controle de peso estruturados no emprego de terapia de modificação do estilo de vida, raramente são alcançadas por jovens com obesidade grave (Ryder, Fox, \& Kelly, 2018). 
Assim, a farmacoterapia, a utilização de dispositivos e a cirurgia bariátrica apresentam potencial capacidade de neutralizar mecanismos orgânicos que favorecem o surgimento e a manutenção da obesidade. A terapia medicamentosa é considerada como uma importante alternativa quando não são alcançados resultados satisfatórios apenas com mudanças de hábitos de vida e nutricionais (Camacho et al., 2019; Ryder et al., 2018).

Nesse contexto, o presente estudo teve como objetivo realizar uma investigação na literatura nacional e internacional sobre as principais intervenções nutricionais e os fármacos mais utilizados para tratamento dessa doença em pediatria, através de uma análise sistemática nas bases eletrônicas de dados. Com relação aos medicamentos antiobesidade, foram pesquisados os principais recomendados pela Sociedade Brasileira de Pediatria (SBP), destacando seus mecanismos de ação, indicações, apresentações, doses e reações adversas, além de suas situações frente à permissão de uso em crianças e adolescentes no Brasil.

\section{Metodologia}

As revisões sistemáticas em saúde são relevantes na compilação de evidências atuais publicadas ligadas à segurança e eficácia das intervenções na área. Dessa maneira, torna-se possível a melhor análise das informações pelo leitor, fornecendo fundamentação mais aprofundada do tema abordado. Nesse contexto, o presente estudo trata-se de uma revisão da literatura, de caráter qualitativa, elaborada conforme as instruções contidas no Preferred Reporting Items for Systematic reviews and MetaAnalyses (PRISMA).

Segundo Liberati et al. (2009), a metodologia PRISMA utiliza uma lista de verificação aplicada no decorrer da revisão, com o intuito de aprimorar os relatos obtidos, por meio das revisões sistemáticas e metanálises elaboradas. Nesta investigação, as bases eletrônicas de dados sistematicamente exploradas para a obtenção de artigos científicos foram Scientific Eletronic Library Online (Scielo), National Library of Medicine (Pubmed) e Sciencedirect.

Em todas as etapas de elaboração da pesquisa, a busca, a seleção, a extração e a análise dos dados foram realizadas aos pares, seguida de discussão entre os autores, que elegeram as publicações mais pertinentes para integrarem o estudo. Entretanto, surgindo qualquer discordância, um terceiro revisor foi consultado para a resolução da possível divergência.

Para a coleta de dados foi utilizada a técnica de documentos, que, de acordo com Pereira, Shitsuka, Parreira e Shitsuka (2018), são procedimentos que auxiliam na operacionalização dos métodos, por meio da busca de artigos publicados em revistas e jornais, contribuindo no resultado final da pesquisa.

Os critérios de inclusão escolhidos para a seleção dos materiais foram: artigos nas categorias original, revisão de literatura, revisão sistemática e meta-análise, bem como o Manual de Orientação da Obesidade na infância e adolescência, da SBP, informações do website da Agência Nacional de Vigilância Sanitária (ANVISA) e estudos da revista Research, Society and Development. As publicações selecionadas foram nos idiomas português e inglês.

Os descritores presentes nos títulos e/ou resumos dos materiais utilizados foram: obesidade pediátrica ou pediatric obesity; tratamento ou treatment; sibutramina ou sibutramine; orlistate ou orlistat; liraglutida ou liraglutide e aleitamento materno ou breastfeeding. As publicações consideradas mais relevantes para o desenvolvimento deste trabalho foram observadas entre os anos de 2003 e 2020, sendo este o intervalo de tempo adotado.

Foram excluídas da pesquisa as publicações que não eram relacionadas com o objetivo tema desta revisão bibliográfica, os publicados nos formatos de trabalho de conclusão de curso, dissertação, tese, relato de caso, resenha e resumo de congresso, bem como em idiomas além do português e do inglês. A partir da leitura dos resumos, foram excluídos também os que não possuíam informações que complementassem o levantamento bibliográfico ou que apresentassem informações repetidas. 


\section{Resultados e Discussão}

A figura 1 elucida as 1641 publicações encontradas nas três bases eletrônicas de dados consultadas, das quais, após seleção e exclusão dos estudos duplicados, 430 trabalhos foram analisados, levando em consideração os critérios de inclusão e de exclusão descritos na metodologia, bem como a relevância das suas informações. Posteriormente a esse estágio, 106 pesquisas foram analisadas com a leitura total do texto e, ao final desta apreciação, 20 artigos foram introduzidos nesta revisão.

Figura 1 - Fluxograma demostrando o processo de seleção de artigos nas bases de dados utilizadas.

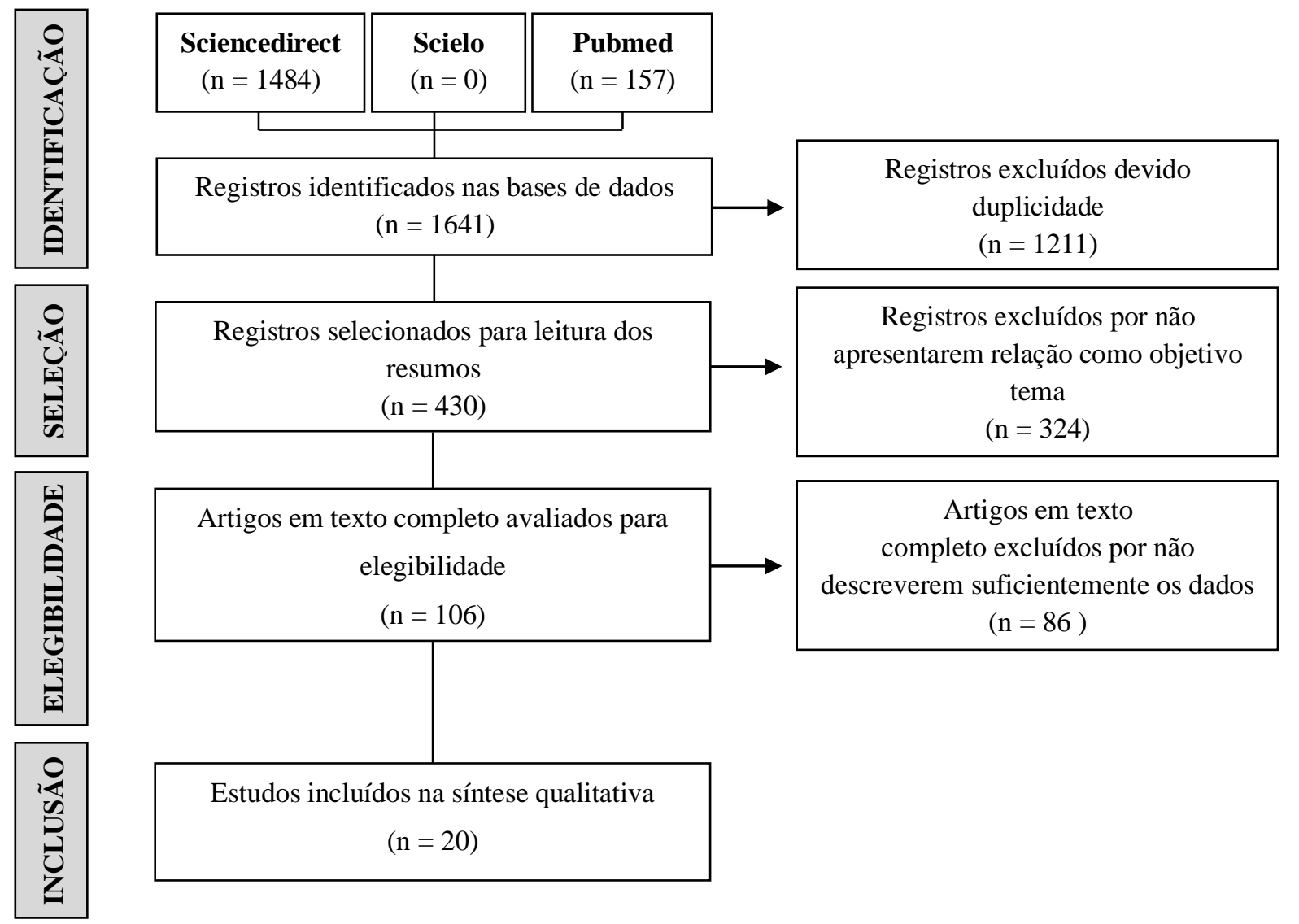

Fonte: Autores.

\subsection{Tratamento nutricional}

As intervenções nutricionais precoces durante a infância são propostas como um meio para evitar que a obesidade se prolongue até a vida adulta, reduzindo o risco de desenvolver comorbidades graves, como síndrome metabólica, diabetes mellitus tipo 2 e doenças cardiovasculares (Kumar et al., 2016; Sanchez, Panahi, \& Tremblay, 2015). Diversos fatores estão envolvidos no desenvolvimento de obesidade infantil, dentre eles, a composição da microbiota, uma dieta com alto teor de açúcar e gordura, e um comportamento sedentário (Kumar \& Kelly, 2017).

Estudos mostram que o consumo de probióticos e prebióticos podem ajudar a modular a microbiota de indivíduos obesos. O Lactobacillus e os gêneros Bifidobacterium são amplamente usados como probióticos, e algumas de suas cepas específicas foram relatadas para melhorar a obesidade e melhorar parâmetros metabólicos (Sivamaruthi, Kesika, Suganthy, \& Chaiyasut, 2019; Yang, Hur, \& Lee, 2017). Além disso, pesquisas confirmam a hipótese de que existem alterações no perfil lipídico de adolescentes com sobrepeso e obesidade, e que essas alterações metabólicas já se fazem presentes muitas vezes 
desde a infância, requerendo uma atenção especial para a necessidade de que haja uma avaliação do perfil lipídico nas consultas de rotina dessa população (Barros et al., 2020).

Outro importante fato a ser considerado é a importância do aleitamento materno enquanto forma de prevenção da obesidade infantil logo na primeira infância. Uma meta-análise demonstrou que a probabilidade de desenvolver obesidade em crianças que nunca foram amamentadas ou que o fizeram por um período menor do que seis meses, era maior do que para aquelas que haviam sido amamentadas por seis meses ou mais (Yan, Liu, Zhu, Huang, \& Wang, 2014).

Deve-se salientar também que, em crianças e adolescentes, as abordagens dietéticas mais flexíveis são melhores utilizadas para apoiar os pacientes e suas famílias a selecionar grupos de alimentos mais saudáveis, a exemplo das fontes de gorduras boas, como, abacate, coco, sementes e oleaginosas que, além de nutritivas, auxiliam no aumento da saciedade, diminuindo as chances de comer em excesso. Adicionalmente, recomenda-se diminuir o tamanho das porções, que não devem ser medidas apenas do ponto de vista quantitativo, mas serem reduzidas de forma progressiva e constante, considerando que a criança está em fase de crescimento e desenvolvimento, e o emagrecimento precisa ser uma consequência de uma reeducação alimentar saudável. Assim, essas medidas proporcionam a redução do excesso de peso de forma mais sustentável, sem restrições alimentares severas (Daniels \& Hassink, 2015).

\subsection{Tratamento farmacológico}

A intervenção farmacológica deve ser realizada de forma muito cautelosa no público pediátrico, sendo indicada, principalmente, quando há comorbidades associadas à obesidade grave, como depressão e compulsão alimentar, dentre outros transtornos psiquiátricos. No Brasil, destacam-se como possibilidades farmacoterapêuticas indicadas pela SBP os medicamentos antiobesidade sibutramina, orlistate e liraglutida (quadro 1) (Mello \& Moretzsohn, 2019).

Quadro 1 - Fármacos antiobesidade que são possibilidades indicadas pela Sociedade Brasileira de Pediatria para o tratamento da obesidade infanto-juvenil.

\begin{tabular}{|c|c|c|c|c|}
\hline Medicamento & Indicação & Apresentação & Dose & $\begin{array}{c}\text { Liberação } \\
\text { no Brasil }\end{array}$ \\
\hline Sibutramina & Indução de saciedade & Cápsulas de $10 \mathrm{a} 15 \mathrm{mg}$ & $\begin{array}{c}10 \mathrm{e} 15 \mathrm{mg} \\
1 \mathrm{vez} \text { ao dia }\end{array}$ & Uso adulto \\
\hline Orlistate & $\begin{array}{c}\text { Bloqueia em } \\
\text { absorção de gordura } \\
\text { no intestino }\end{array}$ & Cápsulas de $120 \mathrm{mg}$ & $\begin{array}{c}120 \mathrm{mg} \\
3 \text { vezes ao dia }\end{array}$ & Uso adulto \\
\hline Liraglutida & $\begin{array}{c}\text { Análogo do peptídeo 1 semelhante } \\
\text { ao glucagon (GLP-1) }\end{array}$ & Injeção subcutânea & $\begin{array}{c}0,6 \mathrm{a} \\
3 \mathrm{mg} / \mathrm{dia}\end{array}$ & $\begin{array}{c}\text { Uso adulto e pediátrico } \\
\text { a partir de } 12 \text { anos }\end{array}$ \\
\hline
\end{tabular}

Fonte: Adaptado de Mello e Moretzsohn (2019).

Para a escolha do agente mais adequado, a equipe de saúde deve avaliar qual melhor opção se adapta à realidade do paciente. Aspectos como vias de administração, posologia, perfil de reações adversas e situação de autorização para uso no país precisam ser considerados no momento da prescrição. O medicamento, para desempenhar sua eficácia terapêutica, precisa ser bem aderido e administrado corretamente e, neste contexto, o cuidador deve ser orientado como proceder, principalmente tratando-se de formulações injetáveis ou que precisem ser ingeridas mais de uma vez ao dia.

Os tratamentos farmacológicos podem desencadear eventos adversos graves e ainda há escassez de estudos que comprovem a segurança terapêutica na pediatria, não sendo, portanto, adequados para todos os pacientes com obesidade 
pediátrica. Apesar disso, quando associado à modificações do estilo de vida, o uso de medicamentos antiobesidade tende a melhorar a indução e a manutenção da perda de peso. Adicionalmente, deve ser considerada a gravidade da obesidade, as tentativas anteriores de perda de peso com ou sem medidas farmacológicas, a idade, a maturação puberal e a presença de outras comorbidades. Assim, os riscos e os benefícios devem ser cuidadosamente analisados pelo prescritor, para a tomada das melhores decisões (Chao et al., 2018).

Outra recomendação relevante, sugerida por Styne et al. (2017), é de que medicamentos para obesidade sejam descontinuados caso não haja redução superior a 4\% no Índice de Massa Corpórea (IMC) após 12 semanas de uso na dosagem completa do medicamento.

Assim, de acordo com os achados na literatura e com as orientações sugeridas pela SBP, os medicamentos mais utilizados, mesmo que alguns de forma off-label, para tratar a obesidade pediátrica, são:

\subsubsection{Sibutramina}

A sibutramina exerce suas ações terapêuticas por meio da inibição da recaptação da noradrenalina, serotonina e dopamina, resultando em aumento da saciedade, viabilizando a redução da ingestão de alimentos, e do gasto energético. Esse fármaco foi avaliado por James et al. (2010) em um importante estudo multicêntrico, o Sibutramine Cardiovascular Outcomes, sendo relatado o elevado risco de reações cardiovasculares graves. Após os resultados, o medicamento foi suspenso na Europa e nos Estados Unido, mas, no Brasil, a substância continua a ser comercializada com criterioso rigor de fiscalização sanitária, com autorização para uso adulto (Franco, Cominato, \& Damiani, 2014; James et al., 2010; Mello \& Moretzsohn, 2019).

Os primeiros ensaios que avaliaram o uso da sibutramina em pacientes obesos adolescentes foram realizados por Berkowitz, Wadden, Tershakovec e Cronquist (2003). Neles, os participantes tinham idades entre 13 e 17 anos, apresentavam IMC de 32 a $44 \mathrm{~kg} / \mathrm{m}^{2}$ e receberam placebo ou sibutramina, associados à terapia comportamental. No $6^{\circ}$ mês de pesquisa, foi verificado que os pacientes tratados com terapia comportamental + sibutramina perderam, em média, 7,8 $\mathrm{kg}(6,3 \mathrm{~kg})$, correspondendo à redução de 8,5\% (6,8\%) do IMC inicial. Já os adolescentes tratados com terapia comportamental + placebo perderam 3,2 kg (6,1 kg), equivalendo a redução de 4,0\% (5,4\%) no IMC (tamanho do efeito, 0,73; intervalo de confiança de $95 \%$ [IC], 0,28-1,18; $\mathrm{p}=0,001)$.

Entretanto, durante os estudos, os participantes do grupo tratado com terapia comportamental + placebo foram trocados no mês 7 para sibutramina, resultando numa perda total de 4,5 $\mathrm{kg}(8,8 \mathrm{~kg})$, o que equivaleu à redução de $6,4 \%(8,3 \%)$ no IMC inicial. Quanto à ocorrência de eventos adversos, foram observadas elevações na pressão arterial e frequência de pulso, bem como na pressão ou frequência de pulso não combinadas, com as diferenças se aproximando da significância $(p=0,06)$. Esses resultados sugerem que a sibutramina, associada à terapia comportamental, aumentou de forma considerável a perda de peso em adolescentes obesos, em relação a terapia comportamental sem adição do fármaco (apenas placebo). Portanto, a sibutramina apresenta efeitos benéficos em adolescentes obesos, mas necessita de monitoramento constante, visto que, assim como em adultos, pode causar eventos cardiovasculares indesejáveis (Berkowitz et al., 2003).

Posteriormente, Berkowitz et al. (2006) realizaram um grande estudo multicêntrico relatando os efeitos da sibutramina em adolescentes obesos. Constatou-se que a adição de sibutramina ao programa de terapia, em pacientes obesos entre 12 e 16 anos de idade, resultou em melhorias estatisticamente significativas no IMC, corroborando com os resultados anteriores. No $12^{\circ}$ mês de pesquisa, a redução média no IMC para terapia comportamental + sibutramina foi de $3,1 \mathrm{~kg} / \mathrm{m}^{2}$, enquanto que para terapia comportamental + placebo foi de $0,3 \mathrm{~kg} / \mathrm{m}^{2}(\mathrm{p}<0,001$ por modelo linear de efeitos mistos). Adicionalmente, o tratamento com sibutramina resultou em melhorias estatisticamente significativamente maiores no peso 
corporal, circunferência da cintura, níveis de triglicerídeos, níveis de lipoproteína de alta densidade (HDL), níveis de insulina e sensibilidade à insulina em comparação com o placebo.

Mais recentemente, Franco et al. (2014) realizaram estudos duplos-cegos, placebo controlado do tipo cross-over, envolvendo pacientes entre 10 e 18 anos, pelo período de 13 meses, sendo os participantes também submetidos à avaliação nutricional, avaliação médica e orientação para a realização de atividade física. Foi relatado que ocorreu uma redução no IMC durante os primeiros 6 meses de análises, correspondendo a 2,2 $\pm 3,0 \mathrm{~kg} / \mathrm{m}^{2}$, no grupo sibutramina, e a 1,2 $\pm 1,7 \mathrm{~kg} / \mathrm{m}^{2}$, no grupo placebo. Após esse período, o tratamento foi invertido em relação ao início das pesquisas, ou seja, o grupo sibutramina passou a receber placebo e vice-versa. Ao final dos 13 meses de investigação, os pacientes que iniciaram com o placebo reduziram o IMC em 2,7 $\pm 2,6 \mathrm{~kg} / \mathrm{m}^{2}$ e, os que iniciaram com sibutramina, reduziram em 1,9 $\pm 3,5 \mathrm{~kg} / \mathrm{m}^{2}(\mathrm{p}=0,5)$. Constatouse que não houve diferença estatística na redução do IMC ao iniciar a pesquisa utilizando a sibutramina ou o placebo.

Adicionalmente, houve a diminuição significativa da circunferência abdominal, com de média 3,7 $\pm 5,0 \mathrm{~cm}$, no grupo sibutramina, e o aumento de 0,6 $\pm 4,9 \mathrm{~cm}$ no grupo placebo, durante o primeiro período do estudo $(\mathrm{p}=0,01)$. Foi demonstrado também que ocorreu a propensão à diminuição das taxas de colesterol total, lipoproteína de baixa densidade (LDL), triglicerídeos, insulina, leptina e proteína $\mathrm{C}$ reativa durante o uso da sibutramina, bem como a tendência ao aumento dos níveis de HDL. Quanto aos eventos adversos, constatou-se com maior frequência a cefaleia e a diarreia no grupo placebo e a cefaleia e a constipação no grupo sibutramina. Esses dados corroboram com os estudos anteriores, indicando que a sibutramina pode ser um forte aliado no tratamento da obesidade em pacientes pediátricos, mas que é necessário um acompanhamento rigoroso em relação à segurança do paciente (Franco et al., 2014).

\subsubsection{Orlistate}

Para o tratamento da obesidade em pediatria, o orlistate é o único medicamento aprovado pela Food and Drug Administration (FDA) nos Estados Unidos, sendo recomendado para jovens de 12 anos ou mais. Já na Europa, a Agência Europeia de Medicamentos (EMA) autoriza esse agente apenas para pessoas com 18 anos de idade ou mais, não sendo recomendado para uso pediátrico. No Brasil, o orlistate é um dos medicamentos antiobesidade mais utilizados na prática clínica, sendo apenas liberado para uso adulto e como uma das possibilidades de farmacoterapia em pacientes pediátricos (Mello \& Moretzsohn, 2019).

O orlistate atua inibindo as lipases gastrointestinais, o que impede a quebra da gordura da dieta, aumentando a sua excreção pelas fezes. A gordura dietética permanece no sistema gastrointestinal e não é absorvida ou digerida, reduzindo a absorção geral das gorduras ingeridas em até 30\%. A dose usual do fármaco é de $120 \mathrm{mg}$ três vezes ao dia, juntamente com refeições contendo componentes gordurosos (Camacho et al., 2019; Chao et al., 2018; Mello \& Moretzsohn, 2019).

Os principais eventos adversos associados ao uso de orlistate compreendem: esteatorreia, escape fecal, dor abdominal e risco de deficiência de vitaminas lipossolúveis. Portanto, devido ao risco de má absorção desses micronutrientes, os pacientes podem receber prescrição de suplementos multivitamínicos. Além disso, o orlistate não deve ser utilizado na existência de doenças disabsortivas intestinais, como doença celíaca, doença inflamatória intestinal e doença de Chron (Mello \& Moretzsohn, 2019; Wickham 3rd \& DeBoer, 2015).

Meta-análises de ensaios clínicos randomizados em crianças e adolescentes com obesidade primária, incluindo estudos de 6 à 12 meses, demonstraram que o orlistate na dose de $120 \mathrm{mg}$, associado à mudanças comportamentais, dieta $\mathrm{e}$ exercícios, apresentou redução média do IMC de $0,83 \mathrm{~kg} / \mathrm{m}^{2}$ (IC de 95\%: 0,47-1,19), em comparação ao placebo. Além disso, não foram identificadas diferenças significativas nos lipídios em jejum, glicose ou insulina entre o orlistate e o placebo. O 
perfil de segurança da droga foi semelhante ao observado em adultos, ou seja, um aumento acentuado nas experiências gastrointestinais desagradáveis, mas com baixo de risco para a saúde (Viner, Hsia, Tomsic, \& Wong, 2010).

Estudos investigaram o uso do orlistate em crianças pré-puberes pelo período de 12 semanas. Foi demonstrado que a droga foi bem aderida pelos participantes, que, inclusive, relataram querer continuar o tratamento após o período de avaliação. Observou-se que os efeitos colaterais gastrointestinais, esperados pelo uso do fármaco, foram leves e toleráveis e, além disso, não foram detectados efeitos negativos no bem-estar psicológico ou físico. Durante a avaliação psicológica, os resultados evidenciaram menor desejo por alimentos com alto potencial engordativo, bem como maior preocupação com a forma corporal e controle oral $(\mathrm{p}=0,011)$. A redução de peso foi, em média, de $4 \mathrm{~kg}(\mathrm{p}=0,016)$ e muito relacionada à diminuição da massa gorda (coeficiente de regressão 0,953, p < 0,01) (Norgren, Danielsson, Jurold, Lötborn, \& Marcus, 2003).

Já em estudos randomizados e duplos-cegos de 54 semanas, envolvendo 539 adolescentes obesos, com idades entre 12 e 16 anos, submetidos também à dieta hipocalórica moderada (30\% de calorias de gordura), exercícios e terapia comportamental, foi verificado que houve a redução no IMC nos pacientes tratados com orlistate e com o placebo durante as primeiras 12 semanas após randomização. Após esse período, o IMC estabilizou-se no grupo orlistate, mas aumentou além da linha de base no grupo placebo. Ao final da pesquisa, o IMC diminuiu $0,55 \mathrm{~kg} / \mathrm{m}^{2}$ com orlistate, mas aumentou $0,31 \mathrm{~kg} / \mathrm{m}^{2}$ com placebo $(p=0,001)$. Em relação aos $15,7 \%$ do grupo de placebo, $26,5 \%$ dos pacientes que tomaram a droga tiveram uma redução de $5 \%$ ou mais no IMC ( $\mathrm{p}=0,005)$ e $4,5 \%$ e $13,3 \%$, respectivamente, tiveram uma diminuição de $10 \%$ ou mais no IMC ( $p=0,002)$ (Chanoine, Hampl, Jensen, Boldrin, \& Hauptman, 2005).

Adicionalmente, foi observado que houve a elevação no peso dos pacientes tratados com fármaco em $0,53 \mathrm{~kg}$ e, nos pacientes tratados com placebo, de 3,14 kg ( $<$ 001). A absortometria radiológica de dupla energia mostrou que essa diferença era explicada por mudanças na massa gorda. A circunferência da cintura reduziu $1,33 \mathrm{~cm}$ no grupo orlistate, mas aumentou $0,12 \mathrm{~cm}$ no grupo placebo ( $\mathrm{p}<0,05$ ), e os eventos adversos gastrointestinais foram considerados leves e moderados, ocorrendo em $9 \%$ a $50 \%$, no grupo orlistate, e em $1 \%$ a 13\%, no grupo placebo. Portanto, os resultados das análises demonstraram que o orlistate melhorou significativamente a redução de peso, em comparação ao placebo, e que o uso do agente em adolescentes não levou a sérios problemas de segurança (Chanoine et al., 2005).

\subsubsection{Liraglutida}

A liraglutida, um análogo do peptídeo 1 semelhante ao glucagon (GLP-1), é um fármaco injetável que atua aumentando as taxas de insulina pós-prandial de maneira dependente de glicose, reduzindo a secreção de glucagon, retardando o esvaziamento gástrico e induzindo a perda de peso através da redução do apetite e da ingestão de calórica. Essa droga, na dose de 3,0 mg, possui aprovação pela FDA, EMA e ANVISA como um auxiliar à terapia de estilo de vida, visando o controle de peso em adultos com obesidade ou sobrepeso (Brasil, 2016; Kelly et al., 2020).

Mais recentemente, em agosto de 2020, a liraglutida passou a ser a primeira droga antiobesidade indicada para uso pediátrico no Brasil, associada ao aconselhamento nutricional e à atividade física, para o controle de peso em adolescentes a partir de 12 anos. O uso do fármaco injetável é realizado de acordo com o peso corporal, que deve ser acima de $60 \mathrm{~kg}$, além do diagnóstico de obesidade (IMC correspondendo a $=30 \mathrm{~kg} / \mathrm{m}^{2}$ para adultos por pontos de corte internacionais) (Brasil, 2020b).

Testes clínicos com liraglutida 3,0 mg, avaliaram o controle de peso em adolescentes puberais $(12 \mathrm{a}<18$ anos de idade) com obesidade. Os pacientes receberam a liraglutida, na dose de 3,0 mg, ou placebo, administrados por via subcutânea, uma vez ao dia, por 56 semanas, seguido de um período de 26 semanas de acompanhamento sem tratamento, sendo submetidos do início ao final do estudo à terapia de estilo de vida. Na semana 56, foi observada a redução no IMC de pelo menos $5 \%$ em 
51 de 113 pacientes no grupo tratado com liraglutida e em 20 de 105 pacientes no grupo tratado com placebo (porcentagem estimada, 43,3\% versus 18,7\%), além de uma redução em IMC de pelo menos $10 \%$ observada em 33 e 9 participantes, respectivamente (porcentagem estimada $26,1 \%$ versus $8,1 \%$ ) (Kelly et al., 2020).

Já no aspecto adesão, foi relatado que os adolescentes aderiram bem à farmacoterapia, sendo $>80 \%$. Os eventos adversos mais evidenciados com o uso de liraglutida foram os gastrointestinais, como náuseas, vômitos e diarreia, sendo reações típicas dos agonistas do receptor de GLP-1, e ocorreram principalmente durante o aumento da dose, tornando-se menos frequentes. Os resultados promissores das pesquisas serviram como base principal para a aprovação da droga em pediatria pela ANVISA (Brasil, 2020b; Kelly et al., 2020).

Anteriormente, Danne et al. (2017) investigaram de forma pioneira o uso da liraglutida em adolescentes com obesidade. Foi relatado que o perfil de tolerabilidade da liraglutida era, geralmente, aceitável. Todos os participantes que receberam o fármaco tiveram, pelo menos, um evento adverso emergente do tratamento (EAET), mas a maioria (96,5\%) destes eram leves. Não houveram EAETs graves e nenhum participante desistiu por causa deles. Os mais frequentes eventos adversos foram do trato gastrointestinal, atingindo $85,7 \%$ dos participantes que receberam a liraglutida. Destes, o mais ocorrido com liraglutida e placebo, foi a dor abdominal.

Outra situação observada foi a hipoglicemia, ocorrendo 12 episódios (em 8 indivíduos) de hipoglicemia no grupo de liraglutida e apenas 2 episódios (em 1 indivíduo) com placebo. Quase metade dos episódios de hipoglicemia relatados com liraglutida ocorreram 10 horas ou mais após a refeição anterior, sugerindo que um jejum prolongado pode contribuir para o evento. Nenhuma hipoglicemia grave foi relatada. Quanto aos achados laboratoriais, não foram observadas alterações clinicamente significativas nas medições hematológicas, bioquímicas (incluindo níveis de calcitonina), hormonais, lipídicos ou urinárias nos grupos liraglutida e placebo. Portanto, os resultados sugerem que a liraglutida resultou em um perfil de segurança e tolerabilidade aceitável em adolescentes com obesidade, sem problemas inesperados em comparação com os já relatados para adultos (Danne et al., 2017).

\section{Conclusão}

Fatores de risco no início da vida da criança, que vão da amamentação até a introdução alimentar, podem influenciar maior adiposidade e risco cardiometabólico no início da adolescência. Estratégias de intervenção nutricional para prevenir a obesidade tardia e o risco cardiometabólico podem ser mais eficazes se visarem simultaneamente os vários fatores modificáveis. Entretanto, quando houver necessidade de utilização de fármacos no tratamento da obesidade pediátrica, é imprescindível a avaliação dos benefícios e riscos. Deve-se verificar qual a opção é a mais adequada em cada caso, levando em consideração a via de administração do fármaco, o perfil de eventos adversos e a adesão terapêutica.

Os estudos em crianças e adolescentes evidenciam relativa eficácia e segurança dos medicamentos sibutramina, orlistate e liraglutida, sendo, este último, recentemente aprovado como o primeiro agente autorizado pela ANVISA para tal indicação em pediatria e, os demais, administrados de maneira off-label. Portanto, a equipe multiprofissional que acompanha o paciente deve priorizar a motivação e as mudanças de hábitos de vida e alimentares, sempre na perspectiva da manutenção dos resultados, visto que o tratamento farmacológico ainda é bastante limitado e deve ser levado em conta que os seus benefícios devem compensar seus riscos a longo prazo.

\section{Referências}

Barros, A. M. G., Visco, D. B., da Silva, R. M. P., da Paiva Alves, S. P., da Silva Simões, M. O., Medeiros, C. C. M., \& de Carvalho, D. F. (2020). Perfil lipídico em crianças com sobrepeso e obesidade: uma revisão integrativa. Research, Society and Development, 9(11), e4349119952-e4349119952. 
Berkowitz, R. I., Fujioka, K., Daniels, S. R., Hoppin, A. G., Owen, S., Perry, A. C., Sothern, M. S., Renz, C. L., Pirner, M. A., Walch, J. K., Jasinsky, O., Hewkin, A. C., \& Blakesley, V. A. (2006). Effects of sibutramine treatment in obese adolescents: a randomized trial. Annals of internal medicine, 145(2), 8190 .

Berkowitz, R. I., Wadden, T. A., Tershakovec A. M., \& Cronquist J. L. (2003). Behavior therapy and sibutramine for the treatment of adolescent obesity: a randomized controlled trial. JAMA, 289(14), 1805-1812.

Brasil. (2016). Liraglutida é aprovada como tratamento auxiliar para o controle do peso em adultos. Obtido em https://www.gov.br/anvisa/ptbr/assuntos/noticias-anvisa/2016/liraglutida-e-aprovada-como-tratamento-auxiliar-para-o-controle-do-peso-em-adultos.

Brasil. (2018). Brasil supera baixo peso infantil, mas número de crianças e adolescentes com sobrepeso e obesidade é preocupante, diz ministra Ideli. Obtido em https://www.gov.br/mdh/pt-br/sdh/noticias/2015/marco/brasil-supera-baixo-peso-infantil-mas-numero-de-criancas-e-adolescentes-com-sobre-peso-eobesidade-e-preocupante-diz-ministra-ideli.

Brasil. (2020a). Data lembra importância de combater a obesidade infantil. Obtido em https://www.gov.br/pt-br/noticias/saude-e-vigilanciasanitaria/2020/06/data-lembra-importancia-de-combater-a-obesidade-infantil.

Brasil. (2020b). Saxenda (liraglutida): nova indicação. Obtido em https://www.gov.br/anvisa/pt-br/assuntos/medicamentos/novos-medicamentos-eindicacoes/saxenda-liraglutida-nova-indicacao.

Camacho, W. J. M., Díaz, J. M. M., Ortiz, S. P., Ortiz, J. E. P., Camacho, M. A. M., \& Calderón, B. P. (2019). Childhood obesity: aetiology, comorbidities, and treatment. Diabetes/metabolism research and reviews, 35(8), e3203.

Chanoine, J. P., Hampl, S., Jensen, C., Boldrin, M., \& Hauptman, J. (2005). Effect of orlistat on weight and body composition in obese adolescents: a randomized controlled trial. JAMA, 293(23), 2873-2883.

Chao, A. M., Wadden, T. A., \& Berkowitz, R. I. (2018). The safety of pharmacologic treatment for pediatric obesity. Expert opinion on drug safety, 17(4), 379-385.

Daniels, S. R., \& Hassink, S. G. (2015). The role of the pediatrician in primary prevention of obesity. Pediatrics, 136(1), e275-e292.

Danne, T., Biester, T., Kapitzke, K., Jacobsen, S. H., Jacobsen, L. V., Petri, K. C. C., Hale, P. M., \& Kordonouri, O. (2017). Liraglutide in an Adolescent Population with Obesity: A Randomized, Double-Blind, Placebo-Controlled 5-Week Trial to Assess Safety, Tolerability, and Pharmacokinetics of Liraglutide in Adolescents Aged 12-17 Years. The journal of pediatrics, 181, 146-153.

Franco, R. R., Cominato, L., \& Damiani, D. (2014). The effect of sibutramine on weight loss in obese adolescents. Arquivos brasileiros de endocrinologia e metabologia, 58(3), 243-250.

James, W. P. T., Caterson, I. D., Coutinho, W., Finer, N., Gaal, L. F. V., Maggioni, A. P., Torp-Pedersen, C., Sharma, A. M., Shepherd, G. M., Rode, R. A., \& Renz, C. L. (2010). Effect of sibutramine on cardiovascular outcomes in overweight and obese subjects. The New England journal of medicine, 363(10), 905917.

Kelly, A. S., Auerbach, P., Barrientos-Perez, M., Gies, I., Hale, P. M., Marcus, C., Mastrandrea, L. D., Prabhu, N., \& Arslanian, S. (2020). A randomized, controlled trial of liraglutide for adolescents with obesity. The New England journal of medicine, 382(22), $2117-2128$.

Kumar, H., Toit, E. d., Kulkarni, A., Aakko, J., Linderborg, K. M., Zhang, Y., Nicol, M. P., Isolauri, E., Yang, B., Collado, M. C., \& Salminen, S. (2016). Distinct patterns in human milk microbiota and fatty acid profiles across specific geographic locations. Frontiers in microbiology, $7,1619$.

Kumar, S., \& Kelly, A. S. (2017). Review of childhood obesity: from epidemiology, etiology, and comorbidities to clinical ass essment and treatment. Mayo clinic proceedings, 92(2), 251-265.

Liberati, A., Altman, D. G., Tetzlaff, J., Mulrow, C., Gøtzsche, P. C., Ioannidis, J. P., Clarke, M., Devereaux, P. J., Kleijnen, J., \& Moher, D. (2009). The PRISMA statement for reporting systematic reviews and meta-analyses of studies that evaluate health care interventions: explanation and elaboration. PLoS Medicine, 6(7), e1000100.

Mello, E. D., \& Moretzsohn, M. (2019). Tratamento Farmacológico da Obesidade. In Sociedade Brasileira de Pediatria. Manual de Orientação: obesidade na infância e adolescência (3rd ed., pp. 118-131). São Paulo: Departamento Científico de Nutrologia.

Norgren, S., Danielsson, P., Jurold, R., Lötborn, M., \& Marcus, C. (2003). Orlistat treatment in obese prepubertal children: a pilot study. Acta paediatrica, 92(6), 666-670.

Pereira, A.S.; Shitsuka, D. M.; Parreira, F. J., \& Shitsuka, R. (2018). Construção e validação de instrumentos e técnicas de coleta de dados. In Colusso, P. R. Metodologia da pesquisa científica (1rd ed., pp. 42-43). Santa Maria: UFSM, Núcleo De Tecnologia Educacional.

Rajjo, T., Mohammed, K., Alsawas, M., Ahmed, A. T., Farah, W., Asi, N., Almasri, J., Prokop, L. J., \& Murad, M. H. (2017). Treatment of Pediatric Obesity: An Umbrella Systematic Review. The journal of clinical endocrinology and metabolism, 102(3), 763-775.

Ryder, J. R., Fox, C. K., \& Kelly, A. S. (2018). Treatment options for severe obesity in the pediatric population: current limitations and future opportunities. Obesity, 26(6), 951-960.

Sanchez, M., Panahi, S., \& Tremblay, A. (2015). Childhood obesity: a role for gut microbiota? International journal of environmental research and public health, 12(1), 162-175.

Sivamaruthi, B. S., Kesika, P., Suganthy, N., \& Chaiyasut, C. (2019). A review on role of microbiome in obesity and antiobesity properties of probiotic supplements. BioMed research international, 2019, 3291367. 
Research, Society and Development, v. 10, n. 1, e60010111797, 2021 (CC BY 4.0) | ISSN 2525-3409 | DOI: http://dx.doi.org/10.33448/rsd-v10i1.11797

Styne, D. M., Arslanian, S. A., Connor, E. L., Farooqi, I. S., Murad, M. H., Silverstein, J. H., \& Yanovski, J. A. (2017). Pediatric obesity-assessment, treatment, and prevention: an endocrine society clinical practice guideline. The journal of clinical endocrinology and metabolism, $102(3)$, 709-757.

Viner, R. M., Hsia, Y., Tomsic, T., \& Wong, I. C. K. (2010). Efficacy and safety of anti-obesity drugs in children and adolescents: systematic review and meta-analysis. Obesity reviews: an official journal of the International Association for the Study of Obesity, 11(8), 593-602.

Wickham 3rd, E. P., \& DeBoer, M. D. (2015). Evaluation and treatment of severe obesity in childhood. Clinical pediatrics, 54(10), 929-940.

World Health Organization. (2020). Obesity and overweight. Retrieved October 1, 2020, from https://www.who.int/news-room/fact-sheets/detail/obesity-andoverweight.

Yan, J., Liu, L., Zhu, Y., Huang, G., \& Wang, P. P. (2014). The association between breastfeeding and childhood obesity: a meta-analysis. BMC public health, 14(1), 1267

Yang, B. G., Hur, K. Y., \& Lee, M. S. (2017). Alterations in gut microbiota and immunity by dietary fat. Yonsei medical journal, 58(6), 1083-1091. 